\title{
Comorbidity of Fibromyalgia in Primary Knee Osteoarthritis: Potential Impact on Functional Status and Quality of Life
}

This article was published in the following Dove Press journal: Open Access Rheumatology: Research and Reviews

\author{
Marwa Yahia Mahgoub (D) \\ Basant Mohamed Elnady (D) \\ Haytham Sayed Abdelkader (D) ${ }^{2}$ \\ Raghdaa Abdelkhaleq \\ Abdelhalem (iD ${ }^{2}$ \\ Waleed Ahmed Hassan' \\ 'Rheumatology, Rehabilitation and \\ Physical Medicine Department, Benha \\ University, Benha, Egypt; \\ ${ }^{2}$ Neuropsychiatry Department, Benha \\ University, Benha, Egypt
}

Background: Knee osteoarthritis (OA) is a painful condition with peripheral and central pain transmission. Fibromyalgia (FM) is the role model of central sensitization of pain perception.

Aim: To assess the frequency of FM in knee OA patients and evaluate the impact of FM on mental health and the quality of life in knee OA patients.

Patients and Methods: A total of 121 female patients were recruited and divided into 3 groups: group I of 59 patients with knee OA only, group II of 32 patients with knee OA and FM, and group III of 30 FM patients. Patients underwent history taking, examination, investigations, and radiological evaluation of both knees. The assessment of visual analog scale (VAS), Pittsburgh Sleep Quality Index (PSQI), Pain Anxiety Symptom Scale Short Form 20 (PASS20), Beck Depression Inventory (BDI-II), and PCASEE questionnaire were done for all patients. Lequesne index of knee OA and radiological Kellgren and Lawrence score severity were done for all OA patients. Fibromyalgia Impact Questionnaire (FIQ) was assessed for all FM patients.

Results: FM was diagnosed in $35.5 \%$ of knee OA patients. Group II patients had higher VAS, PASS-20, PSQI, and lower quality of life than either groups I and III, elevated Lequesne index score than group I, significant correlation between VAS and BMI $(p=0.002)$, Lequesne index score $(p<0.001)$, PASS20 $(p=0.006)$, BDI-II score $(p=0.002)$, and FIQ $(\mathrm{P}<0.001)$, and a negative correlation was found between VAS and physical $(\mathrm{p}<0.001)$, anxiety $(\mathrm{p}=0.046)$, and social $(\mathrm{p}=0.026)$ QoL parameters. Univariable regression analysis showed a higher age, VAS, PSQI, PASS20, and FIQ were associated with lower QoL in group II. A higher PASS20 was the only predictor of lower QoL in multivariable analysis.

Conclusion: Fibromyalgia coexists frequently in knee OA patients and has implications on their mental health, functional activity, and quality of life.

Keywords: knee osteoarthritis, fibromyalgia, central sensitization, osteoarthritis pain

\section{Introduction}

Osteoarthritis (OA) is a widespread musculoskeletal disease that can affect many joints like hips and knees especially in the overweight patients. ${ }^{1}$ It is one of the main aching diseases that relatively affect older individuals. Pain of OA predominately impacts the patients' performance and daily activities. ${ }^{2}$ Although joint pathology is blamed for part of this painful condition, comorbidities may enhance the nociceptive processing and influence the clinical presentations. ${ }^{3}$ Additionally, the pain of $\mathrm{OA}$ is mediated by central and peripheral neurophysiological pathways. ${ }^{4}$
Correspondence: Marwa Yahia Mahgoub Email marwa.yahia@fmed.bu.edu.eg 
Therefore, the optimization of pain relief therapy depends on the proper consideration of pain mechanisms in knee OA. ${ }^{5}$

Fibromyalgia (FM) is a prototypical disease of chronic widespread pain associated with sleep disorders, cognitive dysfunction, fatigue, and depression. ${ }^{6}$ It is considered as an obvious form of central sensitization. ${ }^{7}$ Primary FM is unassociated with underlying disorder while secondary FM occurs in patients with underlying rheumatic or other organic diseases. ${ }^{8}$ Secondary FM is common among inflammatory arthritis patients. ${ }^{9}$ In contrast, primary FM may be a comorbidity with noninflammatory disorders like OA, migraine, and peripheral neuropathy. Both forms impact either the associated or the co-morbid disease outcomes and treatment strategies. ${ }^{10}$

Chronic pain is the prominent feature of OA and FM. Additionally, they share the central sensitization mechanism of pain transmission which is a pain perception mechanism concerned with chronic pain development and maintenance. ${ }^{11} \mathrm{~A}$ state of hyper-reactivity of the nervous system occurs persistently "wind-up". This state lowers the pain threshold and maintains the pain sensation beyond the initial injury healing. ${ }^{12,13}$

Similarly, OA and FM were proved to have psychofunctional implications as sleep disorders, anxiety, and depression. ${ }^{14-17}$ Pain and its peripheral and central sensitization mechanisms concern with these psychological and functional complaints in both diseases. ${ }^{18,19}$

On the other hand, the comorbidity of FM and knee OA was not well studied and reported. This combination may affect the OA therapy as well as FM and their outcomes. So, we aimed to assess the frequency of comorbid FM in Primary knee OA patients and evaluate the impact of this comorbidity on these patients' mental health and quality of life.

\section{Patients and Methods}

This cross-sectional study was conducted in the Rheumatology, Rehabilitation \& Physical Medicine Department, Faculty of Medicine, Benha University, Egypt. Knee OA patients were collected from the Department's outpatient clinic while FM patients were collected also from the outpatients' clinic of Neuropsychiatric Department. The study has been approved by Benha University Research Ethics Committee (the 1964 Declaration of Helsinki and its revisions). Written informed consents in Arabic were obtained from all participants included in the study.

Patients were diagnosed as primary knee OA according to the American College of Rheumatology (ACR) clinical/ radiographic symptomatic criteria of osteoarthritis. ${ }^{20}$ Fibromyalgia was diagnosed by history and physical examination according to Modified 2010/2011ACR Fibromyalgia Diagnostic Criteria. ${ }^{21}$ The exclusion criteria were: patients with a history of OA in joints other than knee joints, secondary OA, KL grade 4 (as they have a more sedentary life which has a direct impact on QoL), arthritis of diseases other than OA, trauma, chronic inflammatory diseases, chronic infection as tuberculosis, chronic diseases as thyroid dysfunction, diabetes, gout, renal and hepatic diseases, malignancies, pregnancy (menstruating patients), smoking, severe cognitive impairment, psychiatric disorders, and neuropathic pain in addition to male gender patients to rule out the variety in the emotional and physical status between male and female genders that may affect their functional status and quality of life.

All patients initially went to a rheumatologic appointment. They were asked about medical history, family history of OA, exercise type and duration/week, and medications. They were subjected to clinical examination especially musculoskeletal examination, Body mass index (BMI) calculation. The Lequesne index of severity for knee OA was used to assess the functional status of the OA patients. It has 3 parameters (Pain or Discomfort, Maximum distance walked, and Activity of daily living). The score is the sum of the points for all parameters with minimum points for each section: 0 , maximum points for each section: 8 , minimum index score: 0 , maximum index score: 24 . The handicap degree (0: none, 1-4: mild, 5-7: moderate, 8-10: severe,11-13: very severe, and $\geq 14$ : extremely severe). ${ }^{22}$ The severity of knee pain was measured by $10 \mathrm{~cm}$ visual analog scale (VAS). ${ }^{23}$

Within the same week, the patients visited the neuropsychiatry team for assessment. The Arabic validated forms of Pain Anxiety Symptom Scale Short Form 20 $(\mathrm{PASS} 20)^{24}$ and the Pittsburgh Sleep Quality Index $(\mathrm{PSQI})^{25}$ questionnaires were used to assess psychological disturbances and sleep quality. The short form of the PASS is a 20-item self-report scale that measures 4 components (cognitive anxiety, escape and avoidance, fear of pain, and physiological anxiety). The frequency of occurrence of each of the 20 behaviors from 0 "never" to 5 "always". ${ }^{24}$ In the PSQI scoring, 7 component scores were derived (Subjective sleep quality, Sleep latency, Sleep duration, Sleep efficiency, Sleep disturbance, use of sleep medications, Daytime dysfunction), each scored 0 (no difficulty) to 3 (severe difficulty). The component 
scores were summed to produce a global score (range 0 to 21). Higher scores indicated worse sleep quality. ${ }^{25}$

The Arabic form of Beck Depression Inventory (BDI-II) was used for the assessment of depression in all patients. ${ }^{26}$ Quality of life (QoL) was assessed using the Arabic form of PCASEE questionnaire which measures the physical, cognitive, affective, social, economic, and ego QoL features. ${ }^{27}$ Each aspect has a final score ranging from 0 to 100 . A detailed evaluation of the socioeconomic status was preformed including collection of data about residence, educational level, marital status, and income. The functional status of FM patients was evaluated using the Arabic validated version of Fibromyalgia Impact Questionnaire (FIQ). ${ }^{28,29}$

Then, radiographs of anteroposterior view in weightbearing position for both knees were obtained from all participants. Kellgren and Lawrence (KL) grading score was used to evaluate the radiological severity of knee OA. $^{30}$

\section{Statistical Methods}

The data were analyzed by SPSS (25.00) Data were presented and suitable analysis was done according to the type of data obtained for each parameter. KolmogorovSmirnov test was done to calculate the normality of data distribution. The significant data were nonparametric.

\section{Descriptive Statistics}

Mean, standard deviation ( \pm SD) were used for parametric numerical data, while median and range were used for non-parametric numerical data. Frequency and percentage were used for non-numerical data.

\section{Analytical Statistics}

Student's $T$ test was used to assess the difference between the two study groups' means. Mann-Whitney test ( $U$-test) was used for a non-parametric variable. Chi-square test was used for qualitative variables. Fisher's exact test was used to examine the relationship between two qualitative variables. Correlation analysis was done to assess the strength of association between two quantitative variables. Logistic regression analysis was used for the prediction of risk factors. Univariate regression examined the effect of a single independent variable on a dependent variable; one variable was analyzed at a time. In multivariate regression, several variables were analyzed together for any possible association or interactions. All tests were 2-sided and a $\mathrm{P}$-value $<0.05$ was considered statistically significant.

\section{Results}

According to the clinical examination, 59 (64.8\%) of the patients had knee-OA only (group I) and 32 (35.2\%) had coexistence of knee OA and FM (group II) and 30 patients with FM (group III). Thirty (32.9\%) of our patients were illiterate, and 61 of them (67.1\%) were from rural areas.

In our study, patients' age showed a significant difference between the three groups being oldest in group I and youngest in group III. BMI was higher in OA patients in either group when compared to that of the isolated FM patients. The severity of knee OA was more in group I than group II. Curiously, Group II had significantly higher VAS, PASS-20, and PSQI compared to group I and group II. Moreover, Lequesne index score was significantly elevated in group II compared to group I. There was no statistical significance of the residence, marital status, educational level, and diseases duration differences between the 3 groups. Regarding the depression issue, there was a difference between the 3 groups with a remarkable higher score of BDI-II questionnaire in group II patients. The quality of life variables is shown in Table 1.

Patients of group II had significantly lower quality of life compared to group I and III patients (0.002 and 0.004, respectively) (Figure 1).

The correlations between Lequesne index score, anxiety, and depression scales with different demographic, clinical, and quality of life parameters in group I and II are shown in Tables 2 and 3. In group II, a positive significant correlation was found between VAS and BMI ( $\mathrm{p}=0.002)$, Lequesne index score $(\mathrm{p}<0.001)$, PASS20 $(\mathrm{p}=0.006)$, BDI-II score $(\mathrm{p}=0.002)$, and FIQ $(\mathrm{P}<0.001)$, while a significant negative correlation was found between VAS and physical $(p<0.001)$, anxiety $(\mathrm{p}=0.046)$, and social $(\mathrm{p}=0.026)$ QoL parameters (Table 2).

Regarding patients with group I, VAS had a significant positive correlation with BMI $(\mathrm{p}=0.03)$ and Lequesne index score $(p<0.001)$, while a significant negative correlation was found between VAS and physical $(\mathrm{p}<0.001)$ and social $(\mathrm{p}<0.001)$ QOL parameters (Table 3).

Regression analysis was conducted for prediction of QoL in all studied cases, using age, BMI, duration, Lequesne index score, VAS, PSQI, PASS20, BDI-II, and FM comorbidity as confounders. Regarding patients of group I, VAS had a significant positive correlation with BMI ( $\mathrm{p}=0.03)$ and Lequesne index score $(\mathrm{p}<0.001)$, while a significant negative correlation was found between VAS and physical $(\mathrm{p}<0.001)$ and social $(\mathrm{p}<0.001)$ QoL parameters (Table 4). 
Table I Baseline Characteristics of Different Groups

\begin{tabular}{|c|c|c|c|c|c|c|}
\hline & Variable & & $\begin{array}{l}\text { KOA } \\
(n=59) \\
\text { Group I }\end{array}$ & $\begin{array}{l}\text { KOA Patients } \\
\text { with FM }(n=32) \\
\text { Group II }\end{array}$ & $\begin{array}{l}\text { FM }(n=30) \\
\text { Group III }\end{array}$ & $P$ value \\
\hline \multirow[t]{5}{*}{ Demographic } & Age (years) & Mean \pm SD & $53.4 \pm 7.2$ & $37.5 \pm 6.9$ & $47.3 \pm 4.6$ & $\begin{array}{l}<0.00 I^{*} \\
\mathrm{pl}<0.00 \mathrm{I}^{*}, \mathrm{p} 2<0.00 \mathrm{I}^{*}, \mathrm{p} 3<0.00 \mathrm{I}^{*}\end{array}$ \\
\hline & BMI & Mean \pm SD & $33.4 \pm 3.1$ & $29.5 \pm 3.9$ & $33.3 \pm 3.4$ & $\begin{array}{l}<0.00 I^{*} \\
\mathrm{pl}<0.00 I^{*}, \mathrm{p} 2<0.00 \mathrm{I}^{*}, \mathrm{p} 3=0.8\end{array}$ \\
\hline & Residence & $\begin{array}{l}\text { (Rural: } \\
\text { Urban) }\end{array}$ & $(34: 25)$ & $(18: 12)$ & $(22: 10)$ & 0.58 \\
\hline & Marital status & $\begin{array}{l}\text { (Married: } \\
\text { not } \\
\text { married) }\end{array}$ & $(49: 10)$ & $(19: 11)$ & $(23: 9)$ & 0.11 \\
\hline & Educational level & $\begin{array}{l}\text { (Literate: } \\
\text { Illiterate) }\end{array}$ & $(39: 20)$ & $(26: 4)$ & $(26: 6)$ & 0.07 \\
\hline \multirow[t]{10}{*}{ Clinical } & $\begin{array}{l}\text { Disease duration } \\
\text { (years) }\end{array}$ & $\begin{array}{l}\text { Median } \\
(\text { IQR) }\end{array}$ & $3(2-5)$ & $2(2-3)$ & $2(I-3.5)$ & 0.07 \\
\hline & \multirow[t]{3}{*}{$\mathrm{KL}$ grades } & I n(\%) & $16(27.1 \%)$ & - & 18 (56.3\%) & \multirow[t]{3}{*}{$0.02 *$} \\
\hline & & II n(\%) & I7(28.8\%) & - & $7(21.9 \%)$ & \\
\hline & & III n(\%) & $26(44.1 \%)$ & - & $7(21.9 \%)$ & \\
\hline & VAS & $\begin{array}{l}\text { Median } \\
(\mathrm{IQR})\end{array}$ & $3(2.0-4.75)$ & $5(3-7)$ & $6(5-8)$ & $\begin{array}{l}<0.00 I^{*} \\
\mathrm{pl}=0.002^{*}, \mathrm{p} 2=0.04^{*}, \mathrm{p} 3<0.00 \mathrm{I}^{*}\end{array}$ \\
\hline & PSQI & Mean \pm SD & $7.5 \pm 1.7$ & $9.1 \pm 2.7$ & $12.5 \pm 2.5$ & $\begin{array}{l}<0.00 I^{*} \\
\mathrm{PI}<0.00 I^{*}, \mathrm{P} 2<0.00 I^{*}, \mathrm{P} 3<0.00 I^{*}\end{array}$ \\
\hline & PASS 20 & Mean \pm SD & $35.9 \pm 8.4$ & $40.5 \pm 8.6$ & $47.8 \pm 10.9$ & $\begin{array}{l}<0.00 \mathrm{I}^{*} \\
\mathrm{pl}=0.02^{*}, \mathrm{p} 2=0.005^{*} \\
\mathrm{p} 3<0.00 \mathrm{I}^{*}\end{array}$ \\
\hline & BDI-II & $\begin{array}{l}\text { Median } \\
(\text { IQR) }\end{array}$ & $5(2-7)$ & $22.5(\mid 8-27)$ & |4(9-17.5) & $\begin{array}{l}<0.00 I^{*} \\
\mathrm{pl}<0.00 \mathrm{I}^{*}, \mathrm{p} 2<0.00 \mathrm{I}^{*}, \mathrm{p} 3<0.00 \mathrm{I}^{*}\end{array}$ \\
\hline & Lequesene score & $\begin{array}{l}\text { Median } \\
\text { (IQR) }\end{array}$ & $5(4-7)$ & - & $7(5.5-12)$ & $<0.001 *$ \\
\hline & FIQ & Mean \pm SD & - & $42.8 \pm 13.2$ & $49.5 \pm 20.7$ & 0.14 \\
\hline \multirow[t]{6}{*}{ Quality of life } & Physical & Mean \pm SD & $49.8 \pm 14.7$ & $62.1 \pm 14.4$ & $47.6 \pm 14.1$ & $\begin{array}{l}<0.00 I^{*} \\
\mathrm{pl}=<0.00 I^{*}, \mathrm{p} 2=<0.00 I^{*}, \mathrm{p} 3=0.5\end{array}$ \\
\hline & Cognitive & Mean \pm SD & $55.6 \pm 15$ & $51.3 \pm 14.8$ & $49.9 \pm 16.6$ & 0.2 \\
\hline & Affective & Mean \pm SD & $53.5 \pm 14.3$ & $47.6 \pm 15.1$ & $34.4 \pm 17.3$ & $\begin{array}{l}<0.00 I^{*} \\
\mathrm{pl}=0.07, \mathrm{p} 2=0.002^{*}, \mathrm{p} 3<0.00 I^{*}\end{array}$ \\
\hline & Social & Mean \pm SD & $50.5 \pm 15.2$ & $55.2 \pm 12.4$ & $45.8 \pm 13.8$ & $\begin{array}{l}0.04 * \\
P I=I 5, p 2=0.006 *, p 3=0.14\end{array}$ \\
\hline & Economic & Mean \pm SD & $55.2 \pm 19.1$ & $60.9 \pm 16.5$ & $54.8 \pm 22.5$ & 0.36 \\
\hline & Ego & Mean \pm SD & $47.8 \pm 16$ & $35.9 \pm 14.9$ & $34.9 \pm 15.9$ & $\begin{array}{l}<0.00 I^{*} \\
\mathrm{pl}=0.00 \mathrm{I}^{*}, \mathrm{p} 2=0.8, \mathrm{p} 3<0.00 \mathrm{I}^{*}\end{array}$ \\
\hline
\end{tabular}

(Continued) 
Table I (Continued).

\begin{tabular}{|l|l|l|l|l|l|l|}
\hline & \multicolumn{2}{|l|}{ Variable } & $\begin{array}{l}\text { KOA } \\
\text { (n=59) } \\
\text { Group I }\end{array}$ & $\begin{array}{l}\text { KOA Patients } \\
\text { with FM (n=32) } \\
\text { Group II }\end{array}$ & $\begin{array}{l}\text { FM (n=30) } \\
\text { Group III }\end{array}$ & P value \\
\hline \multirow{3}{*}{ Medications } & Acetaminophen & $\mathrm{n}(\%)$ & $49(83 \%)$ & $15(50 \%)$ & $20(62.5 \%)$ & $0.004^{*}$ \\
\cline { 2 - 7 } & NSAIDS & $\mathrm{n}(\%)$ & $46(78 \%)$ & $22(73.3 \%)$ & $27(84.4 \%)$ & 0.6 \\
\cline { 2 - 7 } & Antidepressant & $\mathrm{n}(\%)$ & $4(6.8 \%)$ & $27(90 \%)$ & $12(37.5 \%)$ & $<0.00 I^{*}$ \\
\cline { 2 - 7 } & Gabapentinoids & $\mathrm{n}(\%)$ & $9(15.3 \%)$ & $24(80 \%)$ & $16(50 \%)$ & $<0.00 I^{*}$ \\
\cline { 2 - 7 } & Chondroprotectives & $\mathrm{n}(\%)$ & $46(78 \%)$ & $0(0 \%)$ & $21(65.6 \%)$ & 0.3 \\
\hline
\end{tabular}

Notes: PI: between KOA group and FM group; p2: between FM group and KOA patients with FM co-morbidity group; p3: between KOA group and KOA patients with FM co-morbidity group. ${ }^{*} \mathrm{p}<0.05$.

Abbreviations: KOA, knee osteoarthritis; FM, fibromyalgia; BMI, body mass index; KL, Kellgren and Lawrence; VAS, visual analog scale; PSQI, Pittsburgh Sleep Quality Index; PASS20, Pain Anxiety Symptom Scale Short Form 20; BDI-II, Beck Depression Inventory; FIQ, Fibromyalgia Impact Questionnaire; NSAIDS, non-steroidal antiinflammatory drugs.

Linear regression analysis for prediction of factors affecting the functional status denoting by Lequesne index score in all studied knee OA patients using the same parameters. Univariable analysis revealed that higher
BMI, PSQI, BDI-II, and FM presence associated with higher Lequesne index score, while multivariable analysis showed that higher BMI and BDI-II are predictors of lower QoL in all knee OA cases (Table 5).

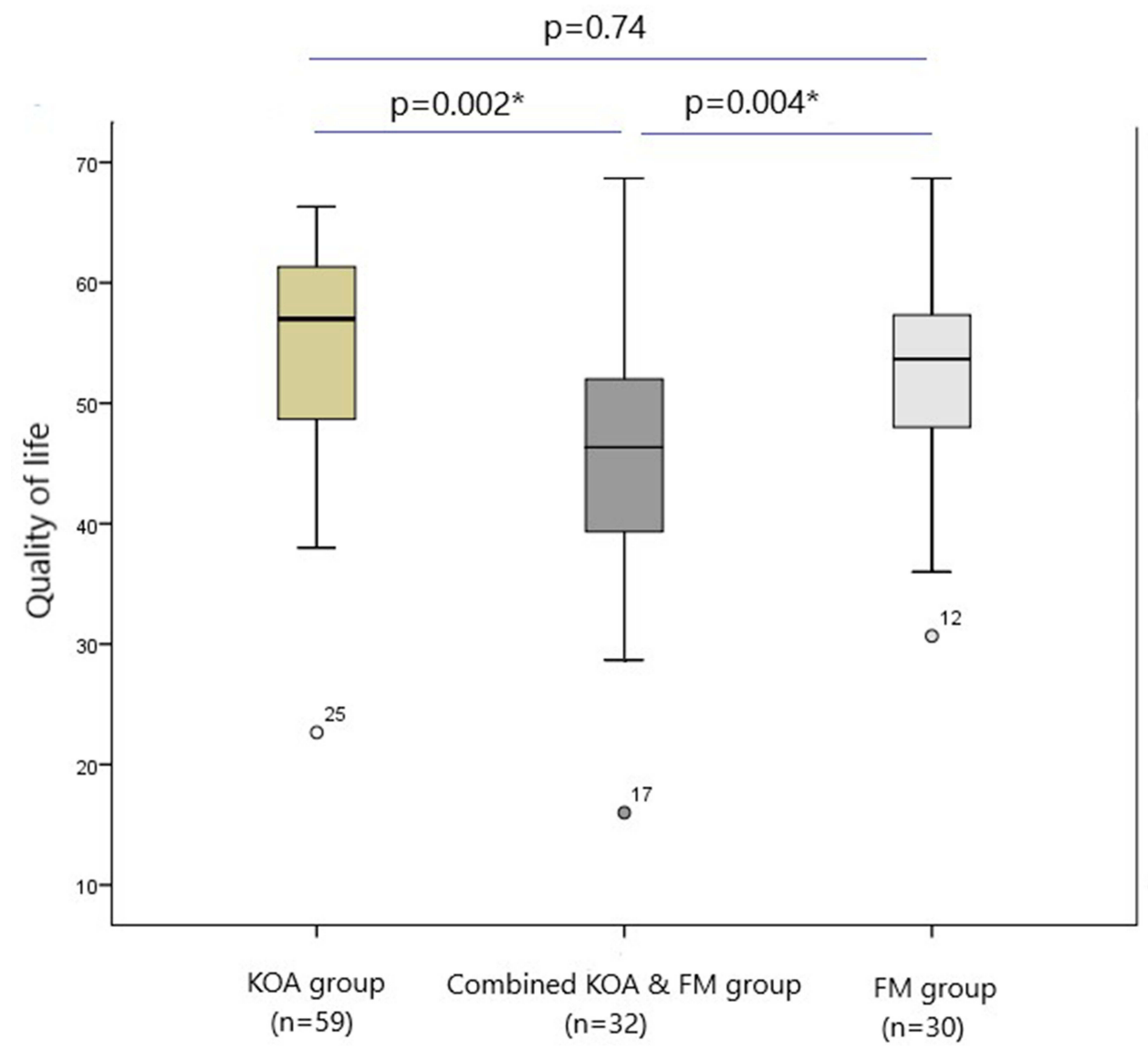

Figure I Quality of life in different groups.

Note: $* P<0.05$.

Abbreviations: KOA, knee osteoarthritis; FM, fibromyalgia. 
Table 2 Correlation of Different Parameters in KOA Patients with FM Comorbidity

\begin{tabular}{|c|c|c|c|c|c|c|c|c|c|}
\hline \multicolumn{2}{|l|}{ Parameters } & \multicolumn{2}{|l|}{ VAS } & \multicolumn{2}{|c|}{ PASS20 } & \multicolumn{2}{|c|}{ BDI-II } & \multicolumn{2}{|c|}{ Lequesne } \\
\hline & & $r$ & $\mathbf{p}$ & $r$ & $\mathbf{p}$ & $r$ & $\mathbf{p}$ & $r$ & $\mathbf{p}$ \\
\hline & $\begin{array}{l}\text { Age } \\
\text { BMI } \\
\text { Disease duration } \\
\text { VAS } \\
\text { PSQI } \\
\text { PASS20 } \\
\text { BDI-II } \\
\text { Lequesne's score } \\
\text { FIQ }\end{array}$ & $\begin{array}{l}0.17 \\
0.53 \\
0.04 \\
- \\
0.23 \\
0.48 \\
0.53 \\
0.64 \\
0.56\end{array}$ & $\begin{array}{l}0.35 \\
0.002^{*} \\
0.84 \\
- \\
0.21 \\
0.006^{*} \\
0.002^{*} \\
<0.001^{*} \\
<0.001^{*}\end{array}$ & $\begin{array}{l}0.46 \\
0.04 \\
0.16 \\
0.48 \\
0.4 \\
- \\
0.25 \\
0.37 \\
0.77\end{array}$ & $\begin{array}{l}0.009^{*} \\
0.84 \\
0.37 \\
0.006^{*} \\
0.02^{*} \\
- \\
0.16 \\
0.04^{*} \\
<0.001^{*}\end{array}$ & $\begin{array}{l}0.13 \\
0.29 \\
0.09 \\
0.53 \\
0.18 \\
0.25 \\
- \\
0.17 \\
0.32\end{array}$ & $\begin{array}{l}0.49 \\
0.11 \\
0.64 \\
0.002 * \\
0.32 \\
0.16 \\
- \\
0.37 \\
0.07\end{array}$ & $\begin{array}{l}0.2 \\
0.38 \\
0.2 \\
0.64 \\
0.28 \\
0.37 \\
0.17 \\
- \\
0.47\end{array}$ & $\begin{array}{l}0.27 \\
0.03^{*} \\
0.26 \\
<0.00 I^{*} \\
0.11 \\
0.04 * \\
0.37 \\
-0.006 *\end{array}$ \\
\hline Quality of life & $\begin{array}{l}\text { Physical } \\
\text { Cognitive } \\
\text { Affective } \\
\text { Social } \\
\text { Economic } \\
\text { Ego }\end{array}$ & $\begin{array}{l}-0.62 \\
-0.1 \\
-0.36 \\
-0.39 \\
0.12 \\
-0.03\end{array}$ & $\begin{array}{l}<0.00 I^{*} \\
0.57 \\
0.046^{*} \\
0.026^{*} \\
0.52 \\
0.88\end{array}$ & $\begin{array}{l}-0.71 \\
-0.47 \\
-0.67 \\
-0.7 \\
-0.11 \\
-0.5\end{array}$ & $\begin{array}{l}<0.00 I^{*} \\
0.007^{*} \\
<0.00 I^{*} \\
<0.00 I^{*} \\
0.56 \\
0.004^{*}\end{array}$ & $\begin{array}{l}-0.4 \\
0.1 \\
-0.1 \\
-0.35 \\
-0.03 \\
0.13\end{array}$ & $\begin{array}{l}0.02 * \\
0.6 \\
0.6 \\
0.049 * \\
0.87 \\
0.49\end{array}$ & $\begin{array}{l}-0.42 \\
-0.09 \\
-0.32 \\
-0.29 \\
-0.04 \\
-0.04\end{array}$ & $\begin{array}{l}0.02 * \\
0.6 \\
0.08 \\
0.1 \\
0.85 \\
0.85\end{array}$ \\
\hline
\end{tabular}

Note: ${ }^{*} \mathrm{p}<0.05$.

Abbreviations: KOA, knee osteoarthritis; FM, fibromyalgia; BMI, body mass index; VAS, visual analog scale; PSQI, Pittsburgh Sleep Quality Index; PASS20, Pain Anxiety Symptom Scale Short Form 20; BDI-II, Beck Depression Inventory; FIQ, Fibromyalgia Impact Questionnaire.

Table 3 Correlation of Different Parameters in Confined KOA Group

\begin{tabular}{|c|c|c|c|c|c|c|c|c|c|}
\hline \multicolumn{2}{|l|}{ Parameters } & \multicolumn{2}{|l|}{ VAS } & \multicolumn{2}{|c|}{ PASS20 } & \multicolumn{2}{|l|}{ BDI-II } & \multicolumn{2}{|c|}{ Lequesne } \\
\hline & & \multirow{2}{*}{$\begin{array}{l}r \\
0.07\end{array}$} & \multirow{2}{*}{$\begin{array}{l}\mathbf{p} \\
0.61\end{array}$} & \multirow{2}{*}{$\begin{array}{l}\boldsymbol{r} \\
-0.2\end{array}$} & \multirow{2}{*}{$\begin{array}{l}\mathbf{p} \\
0.13\end{array}$} & \multirow{2}{*}{$\begin{array}{l}r \\
-0.06\end{array}$} & \multirow{2}{*}{$\begin{array}{l}\mathbf{P} \\
0.63\end{array}$} & \multirow{2}{*}{$\begin{array}{l}r \\
0.07\end{array}$} & \multirow{2}{*}{$\frac{\mathbf{p}}{0.59}$} \\
\hline & Age & & & & & & & & \\
\hline & BMI & 0.3 & $0.03 *$ & 0.03 & 0.84 & 0.36 & $0.005^{*}$ & 0.3 & $0.03^{*}$ \\
\hline & Disease duration & 0.05 & 0.7 & 0.21 & 0.1 & 0.15 & 0.26 & 0.14 & 0.3 \\
\hline & VAS & - & - & 0.05 & 0.71 & 0.11 & 0.39 & 0.74 & $<0.001 *$ \\
\hline & PSQI & 0.05 & 0.73 & 0.38 & $0.003 *$ & 0.33 & $0.0 I^{*}$ & 004 & 0.74 \\
\hline & PASS20 & 0.05 & 0.71 & - & - & 0.27 & $0.04^{*}$ & 0.22 & 0.09 \\
\hline & BDI-II & 0.11 & 0.39 & 0.27 & $0.04 *$ & - & - & 0.02 & 0.85 \\
\hline & Lequesne's score & 0.74 & $<0.001 *$ & 0.22 & 0.09 & 0.02 & 0.85 & - & - \\
\hline \multirow[t]{6}{*}{ Quality of life } & Physical & -0.54 & $<0.001 *$ & -0.31 & $0.02 *$ & 0.06 & 0.67 & -0.65 & $<0.001 *$ \\
\hline & Cognitive & -0.09 & 0.52 & -0.03 & 0.82 & 0.13 & 0.34 & -0.19 & 0.15 \\
\hline & Affective & -0.12 & 0.37 & -0.39 & $0.002 *$ & -0.23 & 0.08 & -0.14 & 0.3 \\
\hline & Social & -0.55 & $<0.001 *$ & -0.34 & $0.009 *$ & 0.1 & 0.42 & -0.6 & $<0.001 *$ \\
\hline & Economic & 0.05 & 0.73 & -0.16 & 0.23 & -0.07 & 0.58 & 0.15 & 0.24 \\
\hline & Ego & -0.18 & 0.17 & -0.25 & 0.06 & -0.16 & 0.24 & -0.3 & $0.02 *$ \\
\hline
\end{tabular}

\footnotetext{
Note: ${ }^{*} \mathrm{p}<0.05$.
}

Abbreviations: KOA, knee osteoarthritis; BMI,body mass index; VAS, visual analog scale; PSQI, Pittsburgh Sleep Quality Index; PASS20, Pain Anxiety Symptom Scale Short Form 20; BDI-II, Beck Depression Inventory.

Regression analysis was conducted for prediction of QoL in group II cases, using age, BMI, duration, Lequesne index score, VAS, PSQI, PASS20, FIQ, and BDI-II as confounders. Higher age, VAS, PSQI, PASS20, and FIQ were associated with lower QoL in univariable analysis. By using significant confounders in Univariable analysis into multivariable analysis revealed that only higher PASS20 was the only predictor of lower QoL (Table 6).

\section{Discussion}

Quality of life (QoL) is defined as a well-being status of persons in several aspects like physical health, psychological health, education, and socio-economic environment. ${ }^{31}$ Physical disorders represent a main impact on QoL especially when concerning with pain. ${ }^{32}$ In chronic musculoskeletal pain disorders as knee osteoarthritis and fibromyalgia, 
Table 4 Linear Regression Analysis for Prediction of Factors Affecting Quality of Life in All Studied KOA Patients

\begin{tabular}{|l|l|l|l|l|}
\hline \multirow{2}{*}{ Variables } & \multicolumn{2}{|l|}{ Univariate } & \multicolumn{2}{l|}{ Multivariate } \\
\cline { 2 - 5 } & P value & $\boldsymbol{\beta}$ & P value & $\boldsymbol{\beta}$ \\
\hline Age & 0.484744 & 1.2234 & & \\
BMI & 0.239142 & 0.11234 & & \\
Disease duration & 0.232511 & 0.3456 & & \\
Lequesne's score & $0.049234^{*}$ & 0.11381 & 3.3787 & -1.2828 \\
VAS & 0.96678 & -2.157 & & \\
PSQI & $0.000272^{*}$ & -1.289 & $0.0334^{*}$ & -1.08364 \\
PASS20 & 0.103467 & -0.6000 & & \\
BDI-II & $0.012142^{*}$ & -0.4292 & 0.8613 & -0.034 \\
FM & $0.00319^{*}$ & -6.886 & 0.05793 & 1.86320 \\
\hline
\end{tabular}

Notes: $*_{p}<0.05 . \beta$ : regression coefficient.

Abbreviations: KOA, knee osteoarthritis; FM, fibromyalgia; BMI, body mass index; VAS, visual analog scale; PSQI, Pittsburgh Sleep Quality Index; PASS20, Pain Anxiety Symptom Scale Short Form 20; BDI-II, Beck Depression Inventory.

Table 5 Linear Regression Analysis for Prediction of Factors Affecting Lequesene's Score in All Studied KOA Patients

\begin{tabular}{|l|l|l|l|l|}
\hline \multirow{2}{*}{ Variables } & \multicolumn{2}{|l|}{ Univariate } & \multicolumn{2}{l|}{ Multivariate } \\
\cline { 2 - 5 } & P value & $\boldsymbol{\beta}$ & P value & $\boldsymbol{\beta}$ \\
\hline Age & 0.60895 & -0.027146 & & \\
BMI & $0.00351 I^{*}$ & 0.33460 & $0.046788105^{*}$ & 0.1720 \\
Disease duration & 0.57293 & 0.1060 & & \\
VAS & 0.36687 & 1.0920 & & \\
PQAI & $0.00867 I^{*}$ & 0.3093 & 0.664619446 & -0.0558 \\
PASS 20 & 0.60 & 0.13076 & & \\
BDI-II & $0.01460 I^{*}$ & 0.1368 & $0.012639246 *$ & -0.13515 \\
FM & $0.000828^{*}$ & 2.5132 & 0.298019997 & 0.957599 \\
\hline
\end{tabular}

Notes: ${ }^{*} \mathrm{p}<0.05$. $\beta$ : regression coefficient.

Abbreviations: KOA, knee osteoarthritis; FM, fibromyalgia; BMI, body mass index VAS, visual analog scale; PSQI, Pittsburgh Sleep Quality Index; PASS20, Pain Anxiety Symptom Scale Short Form 20; BDI-II, Beck Depression Inventory.

the nociceptive pain is sustained by continuing impulse from peripheral tissues, including muscle, ligaments, and joints. ${ }^{33,34}$ As well, in such diseases, central sensitization affects the pain intensity, threshold, and maintenance. ${ }^{35}$ Quality of life, performance, psycho-functional status, and sleep efficiency are parameters intensely affected by knee OA painful sequelae. ${ }^{36}$ These parameters are seemed also to be influenced by FM. ${ }^{37}$ The functional and psychological statuses of patients with FM and some inflammatory arthritis were studied previously. ${ }^{38-40}$ But, the evaluation of these statuses in patients with combined knee OA and FM was not studied yet. This study aimed to reveal the superimposed impacts of the co-incident FM on knee OA patients' symptomatology.
Table 6 Linear Regression Analysis for Prediction of Factors Affecting Quality of Life in Combined KOA and FM Patients

\begin{tabular}{|l|l|l|l|l|}
\hline \multirow{2}{*}{} & \multicolumn{2}{|l|}{ Univariable } & \multicolumn{2}{l|}{ Multivariable } \\
\cline { 2 - 5 } & $\boldsymbol{\beta}$ & $\mathbf{p}$ & $\boldsymbol{\beta}$ & $\mathbf{p}$ \\
\hline Age & -1.020 & $0.023^{*}$ & -0.100 & 0.773 \\
BMI & -0.182 & 0.774 & & \\
Disease duration & -2.052 & 0.112 & & \\
Lequesene's score & -1.078 & 0.074 & & \\
VAS & -2.251 & $0.046^{*}$ & -0.222 & 0.810 \\
PSQI & -1.762 & $0.034^{*}$ & -0.581 & 0.379 \\
PASS20 & -0.382 & $<0.00 I^{*}$ & -0.944 & $<0.00 I^{*}$ \\
FIQ & -0.298 & $0.002^{*}$ & -0.138 & 0.260 \\
BDI-II & -0.317 & 0.363 & & \\
\hline
\end{tabular}

Notes: $*_{p}<0.05$. $\beta$ : regression coefficient.

Abbreviations: KOA, knee osteoarthritis; FM, fibromyalgia; BMI, body mass index; VAS, visual analog scale; PSQI, Pittsburgh Sleep Quality Index; PASS20, Pain Anxiety Symptom Scale Short Form 20; FIQ, Fibromyalgia Impact Questionnaire; BDI-II, Beck Depression Inventory.

We found about $35 \%$ of our knee OA patients to have comorbidity of OA and FM. There is conflicting data in the literature about the prevalence of FM comorbidity in OA patients. Some reports demonstrated relatively lower frequency as $6 \%{ }^{41}$ and $10 \%,{ }^{42}$ while Dzekan et $\mathrm{al}^{43}$ reported a higher prevalence $(22.83 \%)$ of FM among their OA patients. This discrepancy can be attributed to the difference in the sample size, socio-economic status including educational level as the diagnosis of FM is highly subjective. Also, all our subjects were females, known to have an increased prevalence of FM.

We found that patients with comorbid OA-FM had more implications on their QoL that was related to either functional or psychological status. They showed higher scores of the questionnaires evaluating pain, anxiety, sleep disorders, depression, and the functional condition than either patients of confined knee OA or FM. The presence of FM was a predictor factor of both lower functional status and lower QoL. Moreover, the higher FIQ was one of the factors that affect the QoL of these patients negatively.

The management of painful conditions is a serious issue that can lower the burden by increasing the functional status of the patients. ${ }^{44}$ In our study, we stressed on the coexistent fibromyalgia with OA, a degenerative arthritis. Previous studies emphasized on the concomitant fibromyalgia with inflammatory arthritis and its implications on their disease activity and management; as Rheumatoid Arthritis ${ }^{45-47}$ and Systemic Lupus Erythematosus. ${ }^{48,49}$ Staud discussed the shared pain mechanisms in OA, FM, and low back pain. ${ }^{50}$ Neville et al demonstrated the role of central pain processing 
in patients of knee OA who met the FM criteria. ${ }^{51}$ We studied the quality of life with comorbidity of knee OA and FM. Quality of life of OA patients and FM patients was assessed separately in previous studies. ${ }^{42,52,53}$

Mental health is a crucial aspect of all individuals that affect their attitude in dealing with daily stresses. FM patients mostly have a general state of distress in addition to depression that obstacle their lifestyle. ${ }^{54}$ Furthermore, it was proved that OA patients have a reduction in the mental health level with a depression association of poor outcome. ${ }^{55}$ The present results showed the serious psychological and functional drawbacks of associated FM with knee OA and the further burden on this comorbidity patients' mental health. An ending point that may open a new window in the management plan of those patients.

The point of strength in our study is the first orientation about the comorbidity of knee OA and FM impacts on the quality of life and mental health of these patients. Our study limitations were the relatively small sample size and being a single center study done on one ethnic group. We utilized the Arabic form of PCASEE questionnaire which is not the latest. Illiterate people were not excluded from the study and it was reported that educational level may have an impact on pain perception. $^{56}$

We can settle from this research that FM could be a frequent knee OA comorbidity. This comorbidity may facilitate the pain perception and affect the sleep quality, psychological status, functional outcomes, and consequently the quality of life of knee OA patients. Early identification of such association can improve the management plan accordingly with a better outcome. Future longitudinal researches should spot on the FM treatment lines and their effects on knee OA patients' functional and mental status.

\section{Disclosure}

The authors report no funding and no conflicts of interest for this work.

\section{References}

1. Mankin HJ, Brandt KD. Pathogenesis of osteoarthritis. In: Ruddy S, Haris ED, Sledge CB, editors. Kelley's Textbook of Rheumatology. Vol. II. Philadelphia: WB Saunders Company; 2001:1391-1407.

2. Owens C, Conaghan PG. Improving joint pain and function in osteoarthritis. Practitioner. 2016;260(1799):17-20.

3. Mills K, Hübscher M, O'Leary H, et al. Current concepts in joint pain in knee osteoarthritis. Der Schmerz. 2019;33:22-29.

4. Courtney CA, O'Hearn MA, Hornby TG. Neuromuscular function in painful knee osteoarthritis. Curr Pain Headache Rep. 2012;16(6):518524. doi:10.1007/s11916-012-0299-2.
5. O'Neill TW, Felson DT. Mechanisms of osteoarthritis (OA) pain. Curr Osteoporos Rep. 2018;16(5):611-616. doi:10.1007/s11914018-0477-1

6. Geisser ME, Casey KL, Brucksch CB, Ribbens CM, Appleton BB, Crofford LJ. Perception of noxious and innocuous heat stimulation among healthy women and women with fibromyalgia: association with mood, somatic focus, and catastrophizing. Pain. 2003;102:243-250. doi:10.1016/S0304-3959(02)00417-7

7. Wolfe F, Clauw DJ, Fitzcharles MA, et al. The American College of Rheumatology preliminary diagnostic criteria for fibromyalgia and measurement of symptom severity. Arthritis Care Res. 2010;62:600 610. doi: $10.1002 /$ acr. 20140

8. Hench PK. Secondary fibrositis. Am J Med. 1986;81:60-62. doi:10.1016/0002-9343(86)90877-6

9. Branco JC, Bannwarth B, Failde I, et al. Prevalence of fibromyalgia: a survey in five European countries. Semin Arthritis Rheum. 2010;39:448-453. doi:10.1016/j.semarthrit.2008.12.003

10. Bennett RM, Friend R. Secondary fibromyalgia. $J$ Rheumatol. 2019;46(2):127-129. doi:10.3899/jrheum.180611

11. Van Wilgen CP, Keizer D. The sensitization model to explain how chronic pain exists without tissue damage. Pain Manage Nurs. 2012;13:60-65. doi:10.1016/j.pmn.2010.03.001

12. Phillips K, Clauw D. Central pain mechanisms in chronic pain states - maybe it is all in their head. Best Pract Res Clin Rheumatol. 2011;25:141-154. doi:10.1016/j.berh.2011.02.005

13. Curatolo M, Arendt-Nielsen L, Petersen-Felix S. Central hypersensitivity in chronic pain: mechanisms and clinical implications. Phys Med Rehabil Clin N Am. 2006;17:287-302. doi:10.1016/j. pmr.2005.12.010

14. Hawker GA, Gignac MA, Badley E, et al. A longitudinal study to explain the pain-depression link in older adults with osteoarthritis. Arthritis Care Res (Hoboken). 2011;63(10):1382-1390. doi:10.1002/acr.20298

15. Zullig LL, Bosworth HB, Jeffreys AS, et al. The association of comorbid conditions with patient-reported outcomes in veterans with hip and knee osteoarthritis. Clin Rheumatol. 2015;34(8):14351441. doi:10.1007/s10067-014-2707-y

16. Yilmaz H, Karaca G, Demir Polat HA, Akkurt HE. Comparison between depression levels of women with knee osteoarthritis, rheumatoid arthritis, and fibromyalgia syndrome: a controlled study. Turk J Phys Med Rehab. 2015;61:197-202. doi:10.5152/tftrd.2015.87894

17. Melo LF, Da-Silva SL. Análise neuropsicológica de distúrbios cognitivos em pacientes com fibromialgia, artrite reumatoide e lúpus eritematoso sistêmico. Rev Bras Reumatol. 2012;vol.52(2):pp.181188. doi: $10.1590 /$ S0482-50042012000200003

18. Woolf CJ. Central sensitization: implications for the diagnosis and treatment of pain. Pain. 2011;152(3 Suppl):S2-S15. doi:10.1016/j. pain.2010.09.030

19. Mibu A, Nishiyama T, Tanaka K, Manfuku M, Yono S. Difference in the impact of central sensitization on pain-related symptoms between patients with chronic low back pain and knee osteoarthritis. $J$ Pain Res. 2019;12:1757-1765. doi:10.2147/JPR.S200723

20. Altman R, Asch E, Bloch D, et al. Development of criteria for the classification and reporting of osteoarthritis: classification of osteoarthritis of the knee. Arthritis Rheum. 1986;29(8):1039-49.

21. Wolfe F, Egloff N, Häuser W. Widespread pain and low widespread pain index scores among Fibromyalgia-positive cases assessed with the 2010/211 Fibromyalgia criteria. J Rheumatol. 2016;43(9):17431748. doi:10.3899/jrheum. 160153

22. Lequesne M. The algofunctional indices for hip and knee osteoarthritis. J Rheumatol. 1997;24:pp. 779-781.

23. Scott JANE, Huskisson EC. Vertical or horizontal visual analogue scales. Ann Rheum Dis. 1979;38(6):p.560. doi:10.1136/ard.38.6.560

24. Tashani OA, AlAbas OA, Kabil RA, Johnson MI. Psychometric properties of an arabic pain anxiety symptoms Scale-20 (PASS-20) in healthy volunteers and patients attending a physiotherapy clinic. Int J Behav Med. 2017;24:457-472. doi:10.1007/s12529-016-9608-1 
25. Suleiman KH, Yates BC, Berger AM, Pozehl B, Meza J. Translating the Pittsburgh sleep quality index into Arabic. West J Nurs Res. 2010;32(2):250-268. doi:10.1177/0193945909348230

26. Gharib GA. The operational structure of the BDI -II on an Egyptian sample of the university students. Egypt Psychol Assoc. 2000;10(3).

27. Bech P. Rating Scales for Psychopathology, Health Status, and Quality of Life: A Compendium on Documentation in Accordance with the Dsm-Iii-R and Who Systems. Berlin: Springer-Verlag; 1993.

28. Burckhardt CS, Clark SR, Bennett RM. The fibromyalgia impact questionnaire: development and validation. J Rheumatol. 1991;18:728-733.

29. El-Naby MA, Hefny MA, Fahim AE, Awadalla MA. Validation of an adapted arabic version of fibromyalgia syndrome impact questionnaire. Rheumatol Int, 2013;33(10):2561-2567. doi: 10.1007/s00296-013-2779-Z

30. Kellgren JH, Lawrence JS. Radiological assessment of osteo-arthrosis. Ann Rheum Dis. 1957;16(4):494-502. doi:10.1136/ard.16.4.494

31. Barcaccia B. 4 September 2013. "Quality of Life: Everyone Wants It, but What Is It? '”. Forbes/Education Retrieved 10 May 2016.

32. Niv D, Kreitler S. Pain and quality of life. Pain Prac. 2001;1:150 161. doi:10.1046/j.1533-2500.2001.01016.x

33. Gwilym SE, Keltner JR, Warnaby CE, et al. Psychophysical and functional imaging evidence supporting the presence of central sensitization in a cohort of osteoarthritis patients. Arthritis Rheum. 2009;61(9):1226. doi:10.1002/art.24837

34. Staud R. Is it all central sensitization? Role of peripheral tissue nociception in chronic musculoskeletal pain. Curr Rheumatol Rep. 2010;12:448-454. doi:10.1007/s11926-010-0134-X

35. Coderre TJ, Katz J, Vaccarino AL, Melzack R. Contribution of central neuroplasticity to pathological pain: review of clinical and experimental evidence. Pain. 1993;52:259-285. doi:10.1016/0304-3959(93)90161-H

36. Fertelli TK, Tuncay FO. Fatigue in individuals with knee osteoarthritis: its relationship with sleep quality, pain and depression. Pak J Med Sci. 2019;35(4):1040-1044. doi:10.12669/pjms.35.4.383

37. Galvez-Sánchez CM, Duschek S, Reyes Del Paso GA. Psychological impact of fibromyalgia: current perspectives. Psychol Res Behav Manag. 2019;12:117-127. doi:10.2147/PRBM.S178240

38. Gist AC, Guymer EK, Eades LE, Leech M, Littlejohn GO. Fibromyalgia remains a significant burden in rheumatoid arthritis patients in Australia. Int J Rheum Dis. 2018;21:639-646. doi:10.1111/1756-185X.13055

39. Duffield SJ, Miller N, Zhao S, Goodson NJ. Concomitant fibromyalgia complicating chronic inflammatory arthritis: a systematic review and meta-analysis. Rheumatology(Oxford). 2018;57(suppl_3):14531460. doi:10.1093/rheumatology/key075.533

40. Zhao SS, Duffield SJ, Goodson NJ. The prevalence and impact of comorbid fibromyalgia in inflammatory arthritis. Best Pract Res Clin Rheumatol. 2019;33(3):101423. doi:10.1016/j.berh.2019.06.005

41. Haliloglu S, Carlioglu A, Akdeniz D, Karaaslan Y, Kosar A. Fibromyalgia in patients with other rheumatic diseases: prevalence and relationship with disease activity. Rheumatol Int. 2014;34:12751280. doi:10.1007/s00296-014-2972-8

42. Bergman S, Thorstensson C, Andersson M. Chronic widespread pain and its associations with quality of life and function at a 20- year follow-up of individuals with chronic knee pain at inclusion. BMC Musculoskelet Disord. 2019;20(1):592. doi:10.1186/s12891-019-2976-3
43. Dzekan O, Stanislavchuk M. AB1067 Prevalence of fibromyalgia in patients with painful knee OA. Ann Rheum Dis. 2018;77:1644-1645. doi:10.1136/annrheumdis-2018-213511

44. Dueñas M, Ojeda B, Salazar A, Mico JA, Failde I. A review of chronic pain impact on patients, their social environment and the health care system. J Pain Res. 2016;9:457-467. doi: 10.2147/JPR. S105892

45. Kapoor SR, Hider SL, Brownfield A, Mattey DL, Packham JC. Fibromyalgia in patients with rheumatoid arthritis: driven by depression or joint damage? Clin Exp Rheumatol. 2011;29(6 Suppl 69):S88-91.

46. Coury F, Rossat A, Tebib A, et al. Rheumatoid arthritis and fibromyalgia: a frequent unrelated association complicating disease management. J Rheumatol. 2009;36:58-62. doi:10.3899/jrheum.080366

47. Ranzolin A, Brenol JC, Bredemeier M, et al. Association of concomitant fibromyalgia with worse disease activity score in 28 joints, health assessment questionnaire, and short form 36 scores in patients with rheumatoid arthritis. Arthritis Rheum. 2009;61:794-800. doi:10.1002/art.24430

48. Wolfe F, Petri M, Alarcon GS, et al. Fibromyalgia, systemic lupus erythematosus (SLE), and evaluation of SLE activity. $J$ Rheumatol. 2009;36:82-88. doi:10.3899/jrheum.080212

49. Buskila D, Press J, Abu-Shakra M. Fibromyalgia in systemic lupus erythematosus prevalence and clinical implications. Clin Rev Allergy Immunol. 2003;25:25. doi:10.1385/CRIAI:25:1:25

50. Staud R. Evidence for shared pain mechanisms in osteoarthritis, low back pain, and fibromyalgia. Curr Rheumatol Rep. 2011;13(6):513520. doi:10.1007/s11926-011-0206-6

51. Neville SJ, Clauw AD, Moser SE, et al. Association between the 2011 fibromyalgia survey criteria and multisite pain sensitivity in knee osteoarthritis. Clin J Pain. 2018;34(10):909-917. doi:10.1097/ AJP.0000000000000619

52. Leme M, Susan Y, Maurício M, Meneses SR, Marques A. Pain and quality of life in knee osteoarthritis, chronic low back pain and fibromyalgia: a comparative cross-sectional study. Reumatismo. 2019;71:68-74. doi:10.4081/reumatismo.2019.1104

53. Figueiredo Neto EM, Queluz TT, Freire BF. Physical activity and its association with quality of life in patients with osteoarthritis. Rev Bras Reumatol. 2011;51(6):544-549. doi:10.1590/S0482-5004201100060 0002

54. Van Houdenhove B, Egle U, Luyten P. The role of life stress in fibromyalgia. Curr Rheumatol Rep. 2005;7(5):365-370. doi:10. 1007/s11926-005-0021-z

55. Creamer P, Lethbridge-Cejku M, Costa P, Tobin JD, Herbst JH, Hochberg MC. The relationship of anxiety and depression with self-reported knee pain in the community: data from the baltimore longitudinal study of aging. Arthritis Care Res. 1999;12(1):3-7. doi:10.1002/1529-0131(199902)12:1<3::AID-ART2>3.0.CO;2-K

56. Leclerc A, Gourmelen J, Chastang JF, Plouvier S, Niedhammer I, Lanoë JL. Level of education and back pain in France: the role of demographic, lifestyle and physical work factors. Int Arch Occup Environ Health. 2009;82(5):643-652. doi:10.1007/s00420-008-0375-4
Open Access Rheumatology: Research and Reviews

\section{Publish your work in this journal}

Open Access Rheumatology Research and Reviews is an international, peer-reviewed, open access journal publishing original research, reports, editorials, reviews and commentaries on all aspects of clinical and experimental rheumatology in the clinic and laboratory including the following topics: Pathology, pathophysiology of rheumatological diseases; Investigation, treatment and managemen of rheumatological diseases; Clinical trials and novel pharmacological approaches for the treatment of rheumatological disorders. The manuscript management system is completely online and includes a very quick and fair peer-review system, which is all easy to use. Visit http://www.dovepress.com/testimonials.php to read real quotes from published authors. 